\title{
Multi-winding transformer based diode-clamped multi-level inverter
}

\begin{abstract}
In this paper, a new configuration for diode-clamped multilevel inverter based on multiwinding transformer is proposed, described and simulated. The most important difference between this proposed DC-AC-AC structure and basic structure is that in the proposed structure, back-to-back connected outputs of a multi-winding transformer are superseded the capacitors in basic structure. Simulation a 7 levels of proposed configuration shows $10 \%$ THD.
\end{abstract}

Keyword: Multi-winding; Multi-level inverter; Diode-clamped 